\title{
Rhabdomyosarcoma Arising in Teratoma of the Testis
}

\author{
Capt GK Banfield \\ $M B, C h B$, RAMC
}

\section{Lt Col R Brookstein \\ FRCS, RAMC}

Department of Surgical Oncology, The Queen Elizabeth Military Hospital, Stadium Road, Woolwich, London SE18 4QH

Presented to: The Clinical Medicine Section of The Royal Society of Medicine 15 June 1994.

\begin{abstract}
SUMMARY: Rhabdomyosarcoma arising in teratoma of the testis is an exceptionally rare occurrence. The rhabdomyosarcomatous element infers a less favourable prognosis and standard treatment regimes well recognised for the treatment of teratoma of the testis may not be effective.
\end{abstract}

\section{Introduction}

The development of non germ cell malignancy within gonadal or extragonadal teratoma is a rare occurrence. The majority of these tumours are sarcomas and rhabdomyosarcoma is seen predominately. Such tumours may respond poorly to standard Cisplatin based combination chemotherapy.

We report a case of rhabdomyosarcoma arising within a malignant teratoma intermediate of the testis and present a review of the literature.

\section{Case Report}

A 26-year-old officer presented with a 5 month history of gradual, non tender swelling of the right testis. He gave a history of two previous episodes of right sided epididymitis which had settled on antibiotics. There was no history of previous cryptorchidism. On examination the right testis was swollen to $15 \mathrm{~cm}$ in diameter and was smooth, hard and non tender. The swelling failed to transilluminate and there was no associated palpable lymphadenopathy. Ultrasound examination revealed cystic changes in keeping with neoplasm and he went on to right radical orchidectomy. The pre-operative AFP was raised at $14 \mathrm{kU} / \mathrm{L}$ (Ref. range: 0.8 to $6.5 \mathrm{kU} / \mathrm{L}$ ) but the Beta $\mathrm{HCG}$ was within normal limits. The histology demonstrated a mixture of undifferentiated teratomatous and differentiated teratomatous elements with large areas of rhabdomyosarcomatous stroma.

Staging investigations including Chest X-ray and CT scan of the chest and abdomen were unremarkable confirming the presence of stage 1 disease. After consideration it was decided not to offer adjuvant therapy but to maintain surveillance with monthly follow up and tumour markers together with 3 monthly CT scans. At follow up at 8 months there is no sign of recurrence.

\section{Discussion}

Rhabdomyosarcoma is an extremely rare non germ cell malignancy of the testis which may occur in combination with testicular germ cell tumour (1) or as purE rhabdomyosarcoma (2).

Accurate histological diagnosis is important and can b complicated in some instances as the features of sarcomg may resemble those of undifferentiated teratoma Definitive diagnosis in doubtful cases may be made wit $\underline{8}$ immuno-histochemical studies which will be positive for myoglobin in the presence of rhabdomyosarcoma.

The origin of non germ cell malignancies within germ cell tumours is uncertain but may occur by malignant transformation of pre-existing teratomatous elements or from differentiation of totipotential germ cells (3).

The largest series of non germ cell malignancy within germ cell tumours is that of Ulbright et al (3) who found 11 cases either in the primary tumour or recurrence on examination of 269 cases of teratoma. The testis was the site of the primary germ cell tumour in 7 of the cases, the mediastinum in 3 and the retroperitoneum in 1 . The sarcomatous element of 5 of these patients was rhabdomyosarcoma and 4 of these died of their disease despite Cisplatin based combination chemotherapy. The final patient survived after complete surgical excision. In this group, there was no response to Cisplatin based chemotherapy seen in patients with combined teratoma and rhabdomyosarcoma and where total surgical excision was feasible it was considered the treatment of choice.

Terrier-Lacombe et al (4) described a case of rhabdomyosarcoma present within a mature teratoma which developed early metastasis of the purely $\bar{N}$ rhabdomyosarcomatous component despite radical o orchidectomy and right lumboaortic lymph node $\mathbb{0}$ dissection. The patient later faired poorly despite $\mathbb{\omega}^{\circ}$ Cisplatin based chemotherapy. 
Present management plans for testicular cancer are both standardised and successful and expectations of a favourable outcome are high with over $90 \%$ of cases achieving cure (5). Although small in number, men with testicular teratoma containing containing a rhabdomyosarcomatous element would appear to have a poor prognosis by comparison when placed on standard teratoma regimes.

By convention patients with testicular teratoma undergo radical orchidectomy followed by Cisplatin based chemotherapy and restaging in the presence of metastatic spread. Patients with recurrence generally undergo surgical resection followed, if appropriate, by further Cisplatin based chemotherapy. We would recommend that in men with combined teratoma and rhabdomyosarcoma initial management should be as for the teratoma. Remaining or recurring disease requires full histological assessment and in the presence of a significant rhabdomyosarcomatous element, consideration should be given to alternative chemotherapy regimes.

\section{REFERENCES}

1. Nagahara N, Kitamura H, Kanisawa M, IKEDa Shirai K, Matsushita K. A Testicular Teratoma wi Rhabdomyosarcoma and Seminoma. Acta Pathol Jên 1991; 41: 707-711.

2. AleXander F, Pure Testicular Rhabdomyosarcom: Br J Cancer 1968; 22: 498-501.

3. Ulbright TM, LoEhrer PJ, Roth LM, EINHORN LE, Williams SD, Clark SA. The Development of Nogh Germ Malignancies Within Germ Cell Tumoß. Cancer 1984; 54: 1824-1833.

4. Terrier-Lacombe M, Martinez-Madrigal F, Por $\vec{b}$ W, Rahal J, Droz J. Embryonal Rhabdomyosarcoma Arising in a Mature Teratoma of The Testis: A Cage Report. J Urol 1990; 143: 1232-1234.

5. Horwich A, HENDRY WF. Treatment of Testicuär Cancer. Surgery 1993; 11: 505-510. 